\title{
Suppression of Time Delay Signature in Chaotic Semiconductor Lasers with Filtered Optical Feedback
}

\author{
Yuan Wu, 1,2 Bingjie Wang, ${ }^{1,2}$ Jianzhong Zhang, ${ }^{1,2}$ Anbang Wang, ${ }^{1,2}$ and Yuncai Wang ${ }^{1,2}$ \\ ${ }^{1}$ Key Laboratory of Advanced Transducers \& Intelligent Control System, Ministry of Education, Taiyuan 030024, China \\ ${ }^{2}$ Institute of Optoelectronic Engineering, College of Physics \& Optoelectronics, Taiyuan University of Technology, \\ No. 79 Yingze West Street, Taiyuan, Shanxi 030024, China
}

Correspondence should be addressed to Yuncai Wang; wangyc@tyut.edu.cn

Received 15 October 2013; Accepted 13 December 2013

Academic Editor: Hai Yu

Copyright (C) 2013 Yuan Wu et al. This is an open access article distributed under the Creative Commons Attribution License, which permits unrestricted use, distribution, and reproduction in any medium, provided the original work is properly cited.

\begin{abstract}
We report the investigation of the time delay signature in chaotic semiconductor subject to delayed filtered optical feedback, of which a Fabry-Pérot interferometer type of filter is placed in the external cavity. The numerical results show that the suppression of time delay is dependent on the spectral width of the filter and its detuning from the solitary laser frequency. Such laser system will be helpful to acquire high quality chaotic signals.
\end{abstract}

\section{Introduction}

Semiconductor lasers with delay external optical feedback are known to exhibit rich nonlinear dynamical output behavior $[1,2]$. Because of its unique virtues, for example, simple configuration, small size, and feasible controllability, it has been widely applied in chaos-based communication system [3], fast random bit sequences generator [4], chaotic lidar [5] and chaotic optical time-domain relectometry (chaoticOTDR) [6], and so forth. Nevertheless, for conventional optical feedback (COF), the output waveform from the semiconductor laser subject to the mirror feedback often presents the undesirable time delay signatures. The appearance of the time delay signatures in the dynamics compromises the performances of the system. In the context of chaosbased communications, the time delay signature allows an eavesdropper to successfully retrieve the time delay, which, in some systems, is enough to reconstruct the underlying chaotic dynamics [7]. For random bit generation, the time delay signature in the dynamics will significantly affect the statistical performance of the bit sequences [8]. For chaotic lidar and chaotic-OTDR, the peak of the time delay in correlation curve deteriorates the signal-noise-rate and decreases the dynamic range. Therefore, in order to provide the high quality chaotic sources, the elimination of time delay is a critical issue.
Recently, much effort has been devoted to the study of time delay concealment. For COF, the researches show the time delay signature can be concealed by adjusting feedback strength or adding the number of feedback cavities using autocorrelation function and delay mutual information [914]. However, quite recently, the researches propose several new methods and present the time delay signatures that can be easily observed and reconstructed in these scenarios for COF [15-18]. Furthermore, in other generating chaos projects, the time delay signature that can be suppressed, but at the cost of increasing the setup complexity [19-21]. These research activities motivate our effort to develop a novel and simple method for generating time delay signature eliminated chaotic signals.

Like mirror feedback, semiconductor laser with filtered optical feedback (FOF) also can be brought to chaotic behaviors. FOF has become a concerned topic of interest, since it has the potential of control over the laser dynamics via two external parameters, the spectral width of the filter and its detuning from the solitary laser frequency [22-24]. But, to the best of our knowledge, the time delay signature in chaotic semiconductor lasers with FOF has not been investigated.

In this paper, we place a Fabry-Pérot interferometer type of filter feedback in the external cavity. Using autocorrelation function and delay mutual information, we find that the 


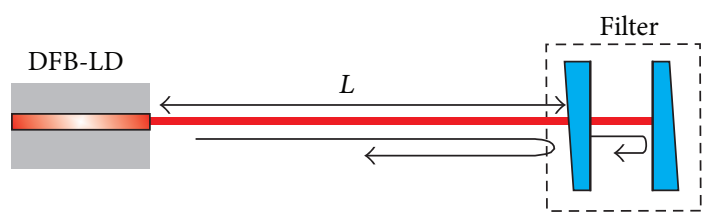

FIGURE 1: Sketch of a semiconductor laser subject to filtered feedback.

suppression of time delay signature is possible in chaotic semiconductor lasers with FOF. The results show that the efficient time delay concealment in FOF mainly depends on the filter linewidth and the frequency detuning from the solitary laser.

\section{Model and Method}

The sketch of a semiconductor laser with FOF is shown in Figure 1. Filtered feedback can be obtained from a grating or a Fabry-Pérot interferometer. In our case we consider a Fabry-Pérot interferometer reflection in FOF. The frequency dependent reflectivity $r(\omega)$ in the FOF is described by a Lorentzian function with filter width (half-width at halfmaximum) $\Lambda$ and detuning $\Delta \omega$ with respect to the solitary laser frequency $\omega_{0}$, that is,

$$
r(\omega)=\frac{\Lambda}{\Lambda+i(\Delta \omega-\omega)}
$$

The distance between the semiconductor laser and filter is the external cavity length $L$, corresponding to the round-trip time of the external cavity $\tau=2 L / c$, which is the time delay signature.

The optical field in the laser is represented by $E(t)=$ $E(t) \exp \left\{i \omega_{0} t\right\}+$ c.c., while $E(t)$ is the (complex) slowly varying amplitude. The laser dynamics in the external feedback with FOF can be described by the set of rate equations [22]:

$$
\begin{aligned}
& \frac{d E(t)}{d t}=\frac{1}{2}(1+i \alpha)\left[\frac{g\left(N(t)-N_{0}\right)}{1+\varepsilon|E(t)|^{2}}-\tau_{p}^{-1}\right] E(t)+\frac{\kappa}{\tau_{\text {in }}} F(t), \\
& \frac{d F(t)}{d t}=\Lambda E(t-\tau) e^{-i \omega_{0} \tau}+(i \Delta \omega-\Lambda) F(t), \\
& \frac{d N(t)}{d t}=\frac{I}{q V}-\frac{N(t)}{\tau_{N}}-\frac{g\left(N(t)-N_{0}\right)}{1+\varepsilon|E(t)|^{2}}|E(t)|^{2},
\end{aligned}
$$

where $\kappa$ denotes the feedback strength of external cavity, $F(t)$ is the (complex) field amplitude reentering from the laser cavity, $N(t)$ is carrier density, and $I$ is pump current. The internal parameters of the laser are considered as follows: transparency carrier density $N_{0}=0.455 \times 106 \mu \mathrm{m} / \mathrm{m}^{3}$, angular frequency of solitary laser $\omega_{0}=1.216 \times 10^{15} \mathrm{rad} / \mathrm{s}$, threshold current $I_{\text {th }}=22 \mathrm{~mA}$, differential gain $g=1.414 \times$ $10^{-3} \mu \mathrm{m}^{-3} \mathrm{~ns}^{-1}$, carrier lifetime $\tau_{N}=2.5 \mathrm{~ns}$, photon lifetime $\tau_{p}=1.17 \mathrm{ps}$, round-trip time in laser intracavity $\tau_{\text {in }}=7.38 \mathrm{ps}$, line-width enhancement factor $\alpha=5.0$, gain saturation parameter $\varepsilon=5 \times 10^{-5} \mu \mathrm{m}^{3}$, and active laser volume $V=$ $324 \mu \mathrm{m}^{3}$.

For a delay-differential system, the time delay signature can typically be retrieved employing either the autocorrelation function (ACF) or the delay mutual information (DMI). The ACF measures how well time series matches its timelagged version, which is defined as

$$
C(\tau)=\frac{\langle(I(t+\tau)-\langle I(t)\rangle)(I(t)-\langle I(t)\rangle)\rangle}{\left(\left\langle(I(t+\tau)-\langle I(t)\rangle)^{2}\right\rangle\left\langle(I(t)-\langle I(t)\rangle)^{2}\right\rangle\right)^{1 / 2}},
$$

where $I(t)$ represents chaotic time series and $\tau$ is the time lag. The delay mutual information (DMI) measures the information shared by two variables, which is based on information theory. The DMI between $I(t)$ and $I(t+\tau)$ is described by

$$
M(\tau)=\sum_{I(t), I(t+\tau)} p(I(t), I(t+\tau)) \log \frac{p(I(t), I(t+\tau))}{p(I(t)) p(I(t+\tau))}
$$

where $p(I(t))$ and $p(I(t+\tau))$ are the marginal probability densities and $p(I(t), I(t+\tau))$ is the joint probability density. The time delay signature can be retrieved from the peak location of ACF or DMI curve.

\section{Numerical Results}

The typical results for FOF and COF with $\tau=5 \mathrm{~ns}$ are comparatively displayed in Figure 2. The left column presents the chaotic time series, power spectrum, and corresponding analysis function curves for the semiconductor laser with mirror feedback for $\kappa=15 \%$ and $I=1.5 I_{\text {th }}$, respectively. The right column presents the corresponding results for the semiconductor laser with Fabry-Pérot interferometer feedback for $\kappa=15 \%$ and $I=1.5 I_{\text {th }}$, the width of filter $\Lambda=15 \mathrm{GHz}$ and frequency detuning $\Delta v=8 \mathrm{GHz}$. From the time series (Figures 2(a1) and 2(a2)), it is very difficult to identify the time delay signature directly since chaotic oscillations behave intricately. However, from the power spectrum (Figure 2(b1)) corresponding to mirror feedback, some uniform spacing peaks emerge upon the background and reveal the external cavity characteristic frequency $0.2 \mathrm{GHz}$. Thus, the value of time delay could be estimated as $5 \mathrm{~ns}$. Meanwhile, from the ACF curve and DMI curve (Figures 2(cl) and 2(d1)), the clear peaks appear at the integer multiples of $\tau$, which reveal the time delay signature. In contrast, for the FOF, the power spectrum (Figure 2(b2)) becomes smooth and has no significant frequency peaks upon background. In addition, no apparent peaks appear in the corresponding ACF and DMI curve (Figures 2(c2) and 2(d2)). These results indicate that the time delay signature of the chaotic signal for the FOF is almost completely suppressed. Compared with mirror feedback, the two special parameters for FOF, the spectral width of the filter and its detuning from the solitary laser frequency, play the critical role in the concealment of time delay signature. 


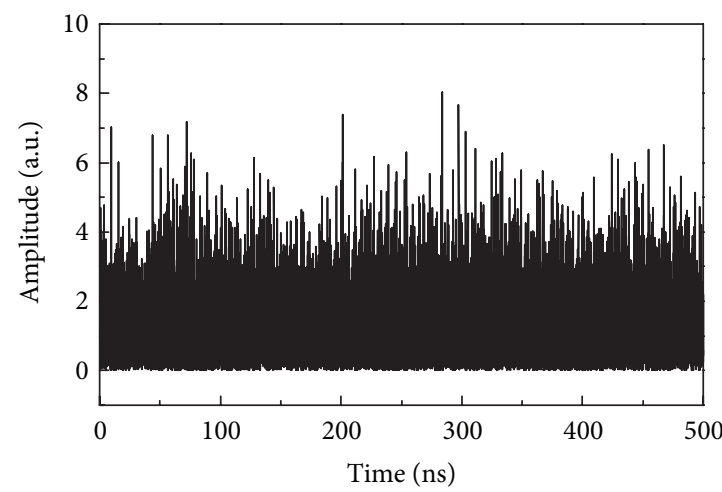

(a1)

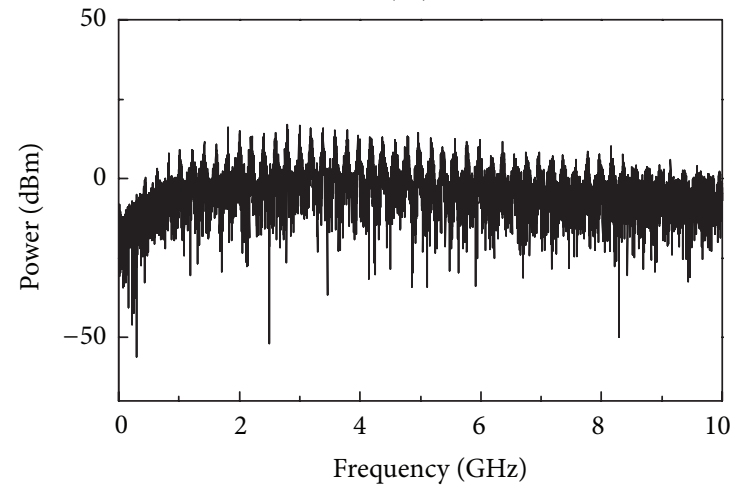

(b1)

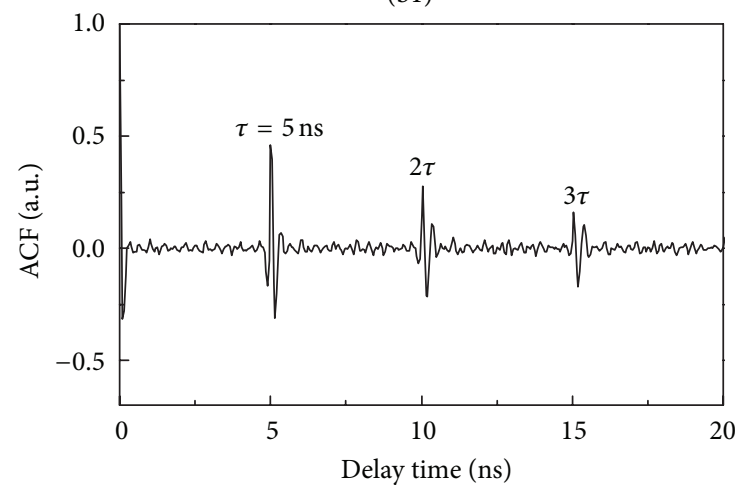

(c1)

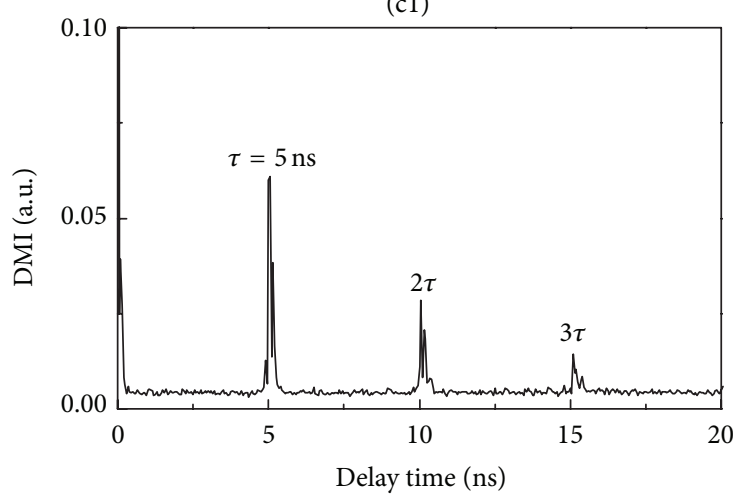

(d1)

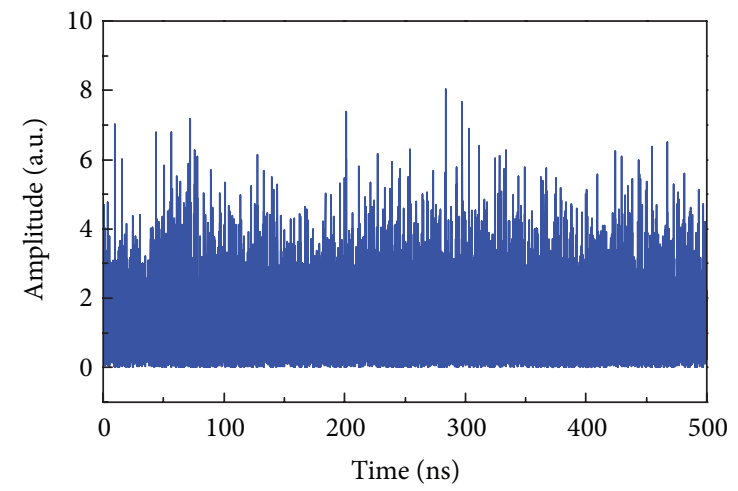

(a2)

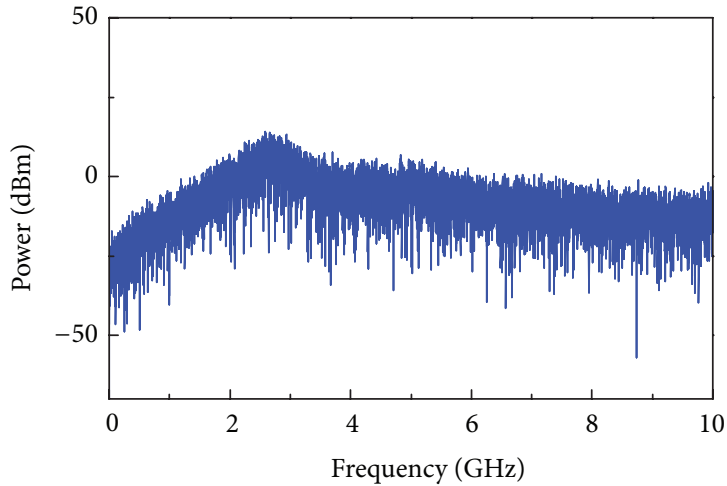

(b2)

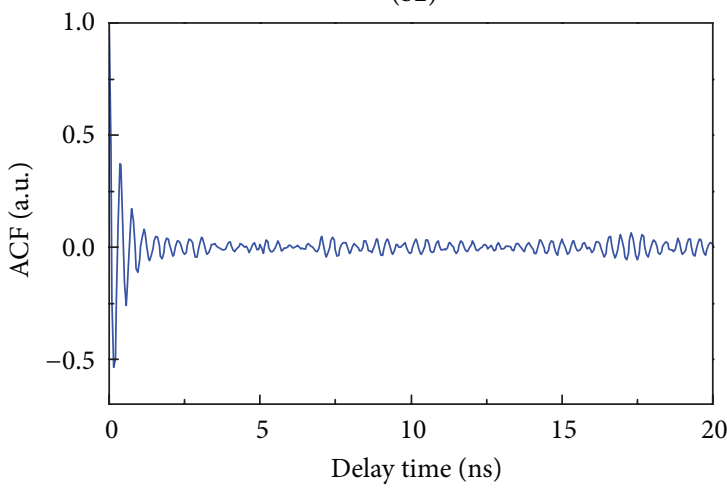

(c2)

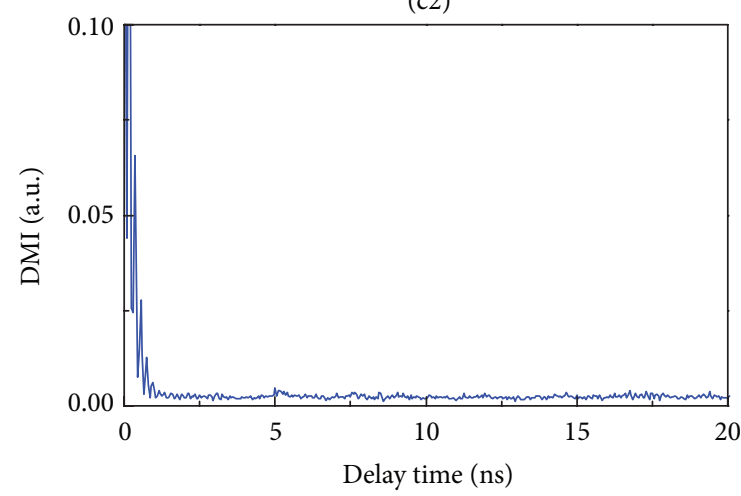

(d2)

FIGURE 2: Chaotic time series, power spectrum, associated ACF curves, and DMI curves for $\kappa=15 \%$ with mirror feedback (left column) and Fabry-Pérot interferometer feedback (right column), respectively. We consider the width of filter $\Lambda=15 \mathrm{GHz}$ and frequency detuning $\Delta v=8 \mathrm{GHz}$. 


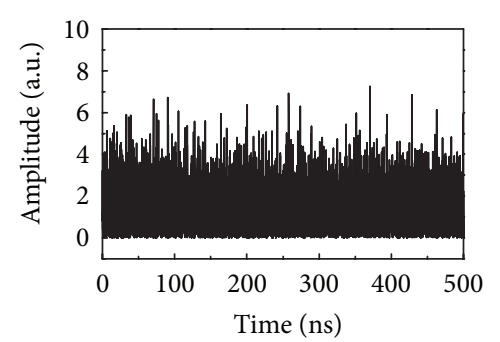

(a1)

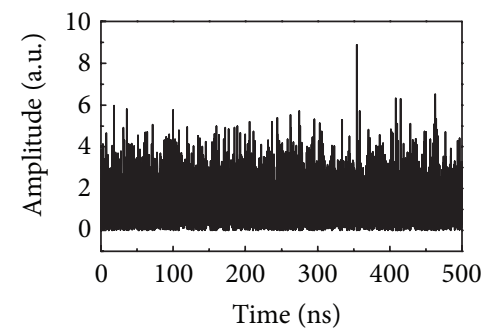

(a2)

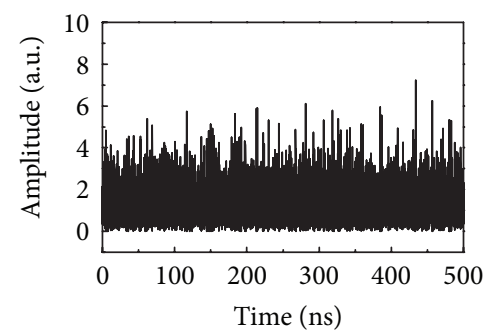

(a3)

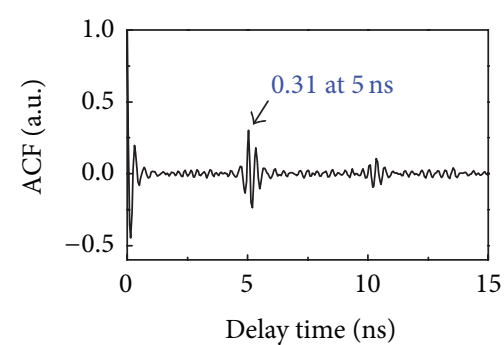

(b1)

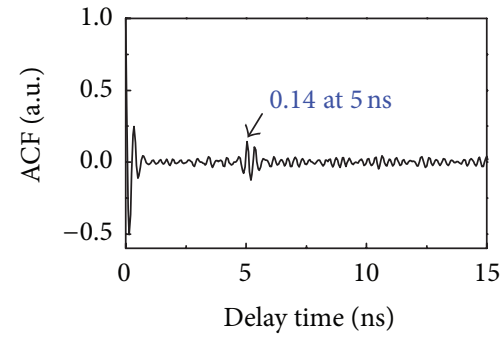

(b2)

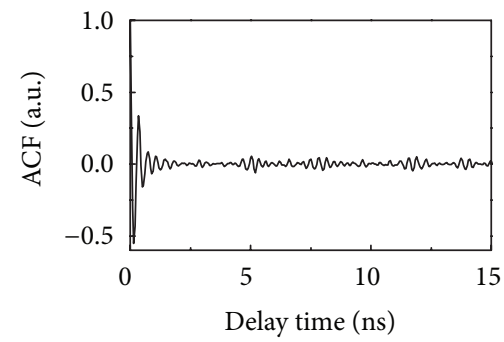

(b3)

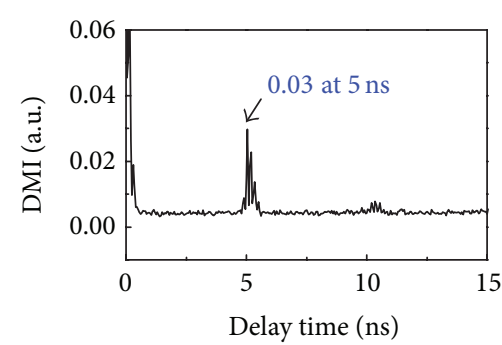

(c1)

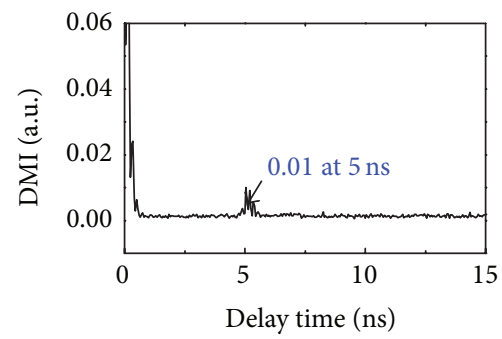

(c2)

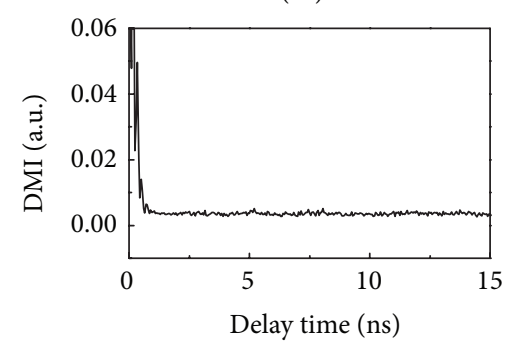

(c3)

FIGURE 3: Time series (a1)-(a3), ACF curves (b1)-(b3), and DMI curves (c1)-(c3) for the different widths of filter, where $\Lambda=17$ GHz (first row), $\Lambda=7 \mathrm{GHz}$ (second row), $\Lambda=3 \mathrm{GHz}$ (third row) keeping time delay $\tau=5 \mathrm{~ns}$, frequency detuning $\Delta \nu=0$, and $\kappa=15 \%$.

Next, we investigate the influence of filter width and frequency detuning on the possibility of the identification of time delay signature. Firstly, we maintain feedback strength $\kappa=15 \%$ and frequency detuning $\Delta \nu=0$ and adjust filter width. We show the time series, ACF curves, and DMI curves for different filter width in Figure 3. From Figures 3(b1) and $3(\mathrm{cl})$, we observe that the time delay signature is clearly exhibited when $\Lambda=17 \mathrm{GHz}$. Combining all ACF and DMI curves, we notice the amplitudes of time delay signature in ACF and DMI are gradually suppressed as $\Lambda$ decrease from 17 to $3 \mathrm{GHz}$. Particularly, when the width decreases to $3 \mathrm{GHz}$ in third row (Figures 3(b3) and 3(c3)), the TD signature is almost shielded completely into the background fluctuations. Furthermore, we show the evolution tendency of time delay signature amplitude in ACF with varying filter widths $\Lambda$ from $4 \mathrm{GHz}$ to $41 \mathrm{GHz}$ in Figure 4 . We observe the amplitude gradually decrease with decreasing $\Lambda$.

In what follows, we investigated the influence of the frequency detuning $\Delta v$ between the filter and the solitary laser on the suppression of the time delay signature. Keeping the width of filter $\Lambda=17 \mathrm{GHz}$, the time series, ACF and DMI curves were presented in Figure 5 when $\Delta v$ varies from $-12 \mathrm{GHz}$ to $14 \mathrm{GHz}$. At first, for $\Delta v=-12 \mathrm{GHz}$ as shown in Figures 5(al)-5(cl), obvious peaks are observed in both $\mathrm{ACF}$ and DMI curves, but the time location corresponding

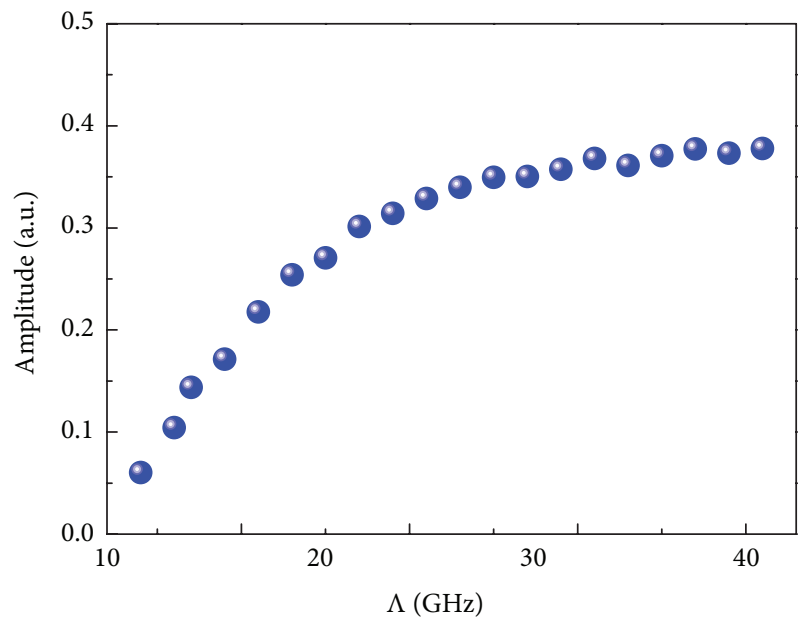

FIGURE 4: The variation of amplitude in ACF with the width of filter keeping time delay $\tau=5 \mathrm{~ns}$, frequency detuning $\Delta v=0$ and, $\kappa=$ $15 \%$.

to peak is $5.5 \mathrm{~ns}$, which deviates from the expected delay time $\tau$. This phenomenon may provide a pseudo time delay signature. Further adjusting $\Delta v$ to $-2 \mathrm{GHz}$, obvious time delay signature can be properly identified from ACF and DMI 


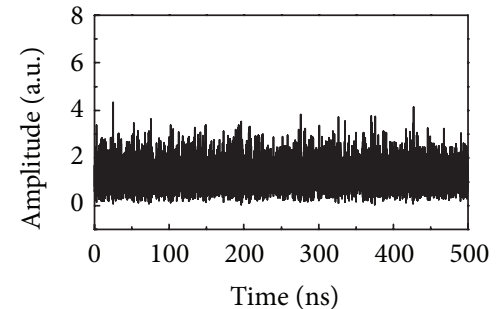

(a1)

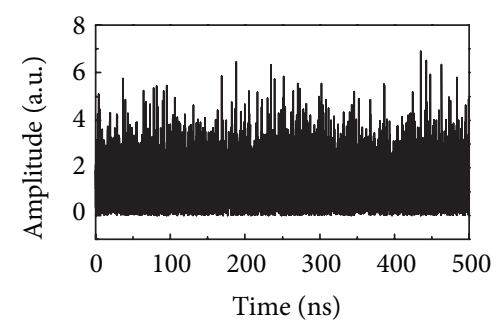

(a2)

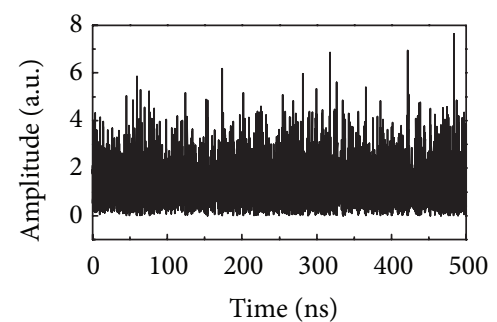

(a3)

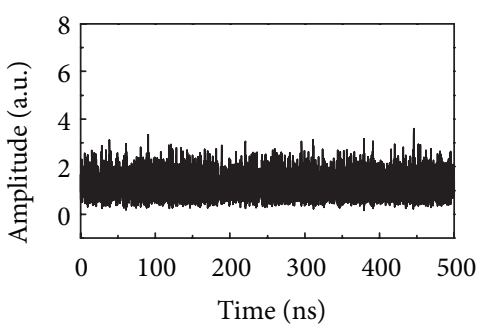

(a4)

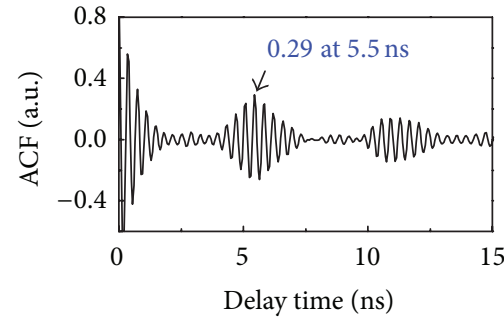

(b1)

(a)

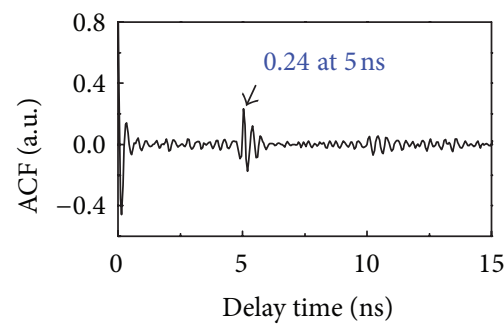

(b2)

(b)

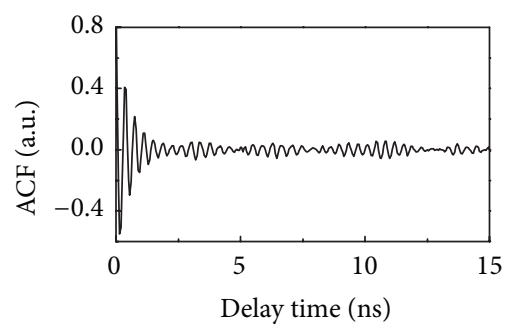

(b3)

(c)

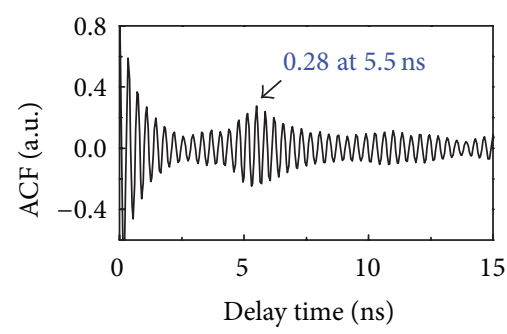

(b4)

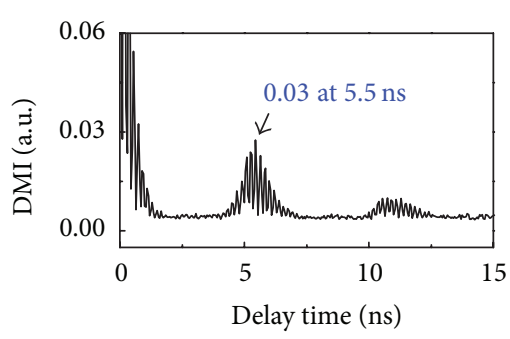

(c1)

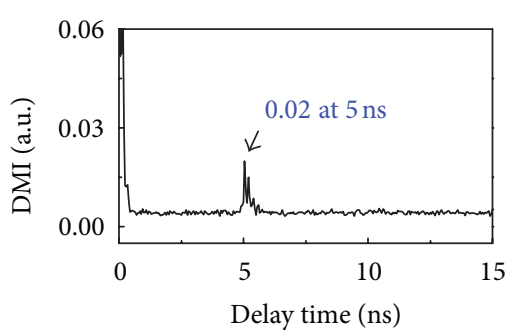

(c2)

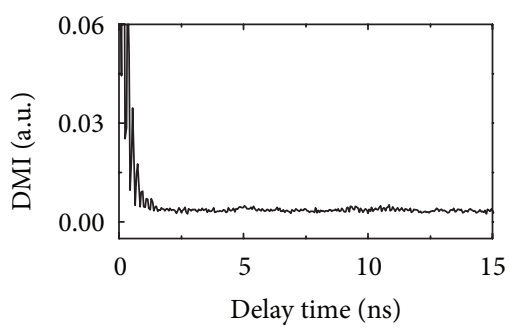

(c3)

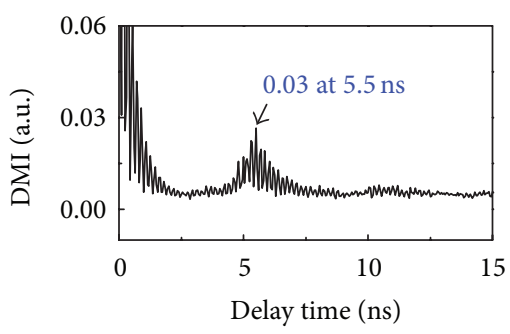

(c4)

(d)

FIgure 5: Time series (a1)-(a4), ACF curves (b1)-(b4), and DMI curves (c1)-(c4) for the different frequency detuning, where $\Delta v=-12 \mathrm{GHz}$ (first row), $\Delta v=-2 \mathrm{GHz}$ (second row), $\Delta \nu=8 \mathrm{GHz}$, (third row), and $\Delta \nu=14 \mathrm{GHz}$ (fourth row) keeping time delay $\tau=5 \mathrm{~ns}$, width $\Lambda=17 \mathrm{GHz}$, and $\kappa=15 \%$.

curves. When $\Delta v$ is turned to $8 \mathrm{GHz}$, the peak amplitude is significantly suppressed close to the background in ACF and DMI curves as shown in Figures 5(a3)-5(c3). In this situation, an eavesdropper would be quite difficult to accurately identify the time delay of the semiconductor dynamical system. However, increasing $\Delta v$ to $14 \mathrm{GHz}$ (Figures $5(\mathrm{a} 4)-5(\mathrm{c} 4)$ ), the amplitudes of ACF and DMI boom again and the time location of peak also deviates again from the expected delay $\tau$. Thus, adjusting the frequency detuning $\Delta v$, not only the amplitude in ACF or DMI for $\tau$ is influenced, but also the time location of peak is affected.

To further show the evolution patter of time delay signature under different frequency detuning $\Delta v$, the variation curves of amplitude and the location of the ACF peak with $\Delta v$ in a time window of $1.0 \mathrm{~ns}$ around $\tau$ are shown in Figures 6(a) and 6(b), respectively. From Figure 6, the evolution of time delay could be roughly divided into three regions. For $-15 \mathrm{GHz}<\Delta v<-4 \mathrm{GHz}$, although the peak 


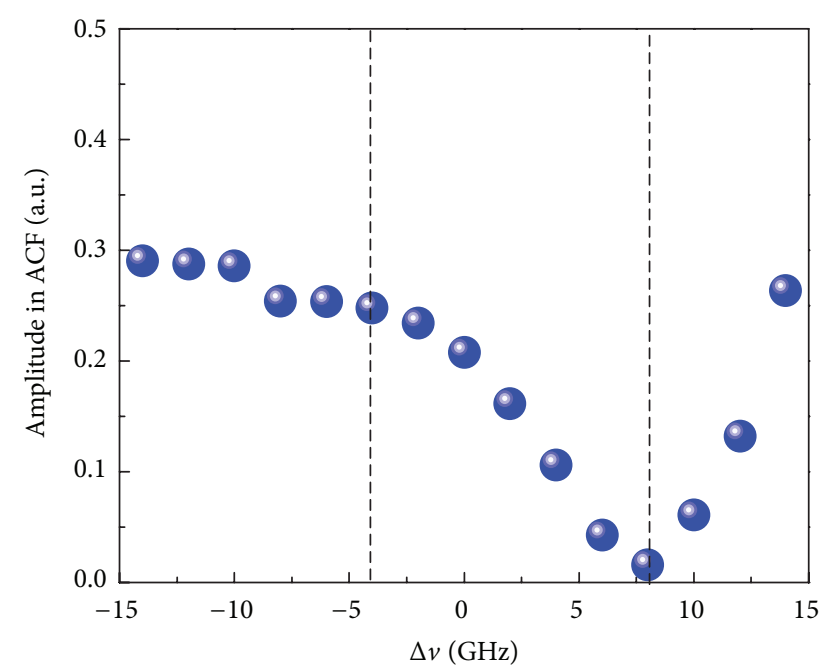

(a)

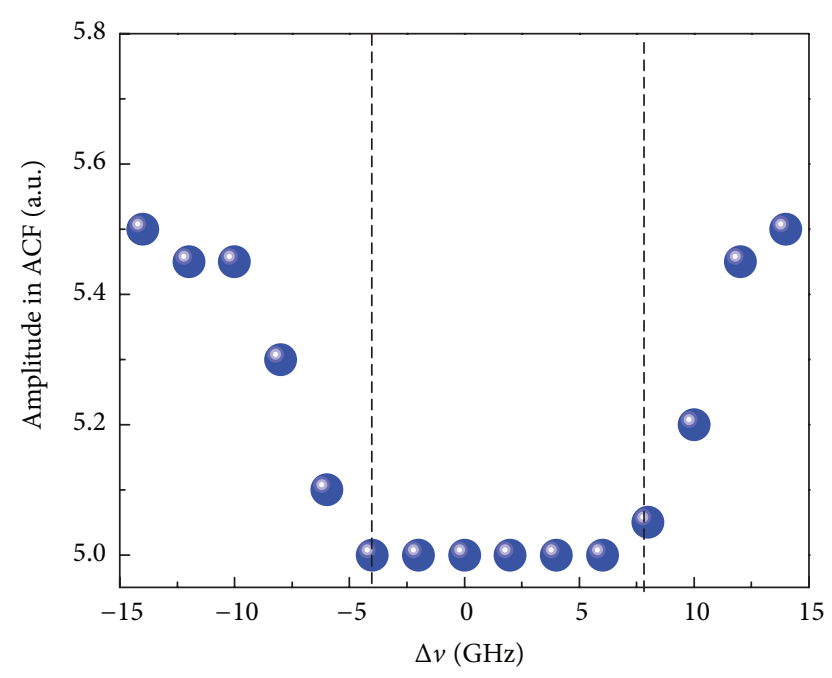

(b)

FIGURE 6: The variation of amplitude and time location in ACF with the frequency detuning $\Delta \nu$ are shown in (a) and (b), respectively.

is obvious, the time location corresponding to peak deviates from the expected $\tau$, so the time delay signature cannot be properly identified. For $-4 \mathrm{GHz} \leqslant \Delta v<8 \mathrm{GHz}$, the time location corresponding to peak is the expected $\tau$, but the amplitude of the time delay signature attenuates significantly with increasing $\Delta v$. When the peak amplitude in ACF is less than 0.1, the time delay signature is almost shielded completely into the background fluctuations. Particularly, when $\Delta v=8 \mathrm{GHz}$, the amplitude decreases to the minimum. For $8 \mathrm{GHz}<\Delta v<15 \mathrm{GHz}$, although the peak booms again, the deviation between time location of the peak and the expected $\tau$ again becomes large with increasing $\Delta \nu$. This phenomenon may provide a pseudo time delay signature. Therefore, one can reasonably deduce that the time delay suppression owns certain tolerance to frequency detuning $\Delta v$ under specific filter width.

\section{Conclusion}

In conclusion, we have demonstrated the time delay signature elimination phenomenon in a semiconductor laser with FOF, which consists of a Fabry-Pérot interferometer type of filter in the external cavity. We find that the time delay signature can be concealed in chaotic semiconductor lasers with FOF under proper filtered width and frequency detuning in terms of autocorrelation function and delay mutual information. We found the scenarios for efficient time delay concealment that would be helpful to acquire high quality chaotic signals in some applications.

\section{Conflict of Interests}

The authors declare no competing financial interests.

\section{Acknowledgments}

This work was supported in part by the National Natural Science Foundation (NSFC) of China under Grants 61227016, 61205142, and 61240017, the Natural Science Foundation of Shanxi Province under Grants 2013011019-3 and 20120210132 , and the Program for the Outstanding Innovative Teams of Higher Learning Institutions of Shanxi, China (OIT).

\section{References}

[1] R. Vicente, J. Daudén, P. Colet, and R. Toral, "Analysis and characterization of the hyperchaos generated by a semiconductor laser subject to a delayed feedback loop," IEEE Journal of Quantum Electronics, vol. 41, no. 4, pp. 541-548, 2005.

[2] A. Wang, Y. Wang, and H. He, "Enhancing the bandwidth of the optical chaotic signal generated by a semiconductor laser with optical feedback," IEEE Photonics Technology Letters, vol. 20, no. 19, pp. 1633-1635, 2008.

[3] A. Argyris, D. Syvridis, L. Larger et al., "Chaos-based communications at high bit rates using commercial fibre-optic links," Nature, vol. 438, no. 7066, pp. 343-346, 2005.

[4] A. Uchida, K. Amano, M. Inoue et al., "Fast physical random bit generation with chaotic semiconductor lasers," Nature Photonics, vol. 2, no. 12, pp. 728-732, 2008.

[5] F.-Y. Lin and J.-M. Liu, "Chaotic lidar," IEEE Journal of Selected Topics in Quantum Electronics, vol. 10, no. 5, pp. 991-997, 2004.

[6] Y. C. Wang, B. J. Wang, and A. B. Wang, "Chaotic correlation optical time domain reflectometer utilizing laser diode," IEEE Photonics Technology Letters, vol. 20, no. 19, pp. 1636-1638, 2008.

[7] S. Ortín, J. M. Gutiérrez, L. Pesquera, and H. Vasquez, "Nonlinear dynamics extraction for time-delay systems using modular neural networks synchronization and prediction," Physica A, vol. 351, no. 1, pp. 133-141, 2005.

[8] J. Zhang, Y. Wang, L. Xue et al., "Delay line length selection in generating fast random numbers with a chaotic laser," Applied Optics, vol. 51, no. 11, pp. 1709-1714, 2012.

[9] D. Rontani, A. Locquet, M. Sciamanna, and D. S. Citrin, "Loss of time-delay signature in the chaotic output of a semiconductor laser with optical feedback," Optics Letters, vol. 32, no. 20, pp. 2960-2962, 2007.

[10] D. Rontani, A. Locquet, M. Sciamanna, D. S. Citrin, and S. Ortin, "Time-delay identification in a chaotic semiconductor 
laser with optical feedback: a dynamical point of view," IEEE Journal of Quantum Electronics, vol. 45, no. 7, pp. 879-891, 2009.

[11] J.-G. Wu, G.-Q. Xia, X. Tang et al., “Time delay signature concealment of optical feedback induced chaos in an external cavity semiconductor laser," Optics Express, vol. 18, no. 7, pp. 6661-6666, 2010.

[12] J.-G. Wu, G.-Q. Xia, and Z.-M. Wu, "Suppression of time delay signatures of chaotic output in a semiconductor laser with double optical feedback," Optics Express, vol. 17, no. 22, pp. 20124-20133, 2009.

[13] M. W. Lee, P. Rees, K. A. Shore, S. Ortin, L. Pesquera, and A. Valle, "Dynamical characterisation of laser diode subject to double optical feedback for chaotic optical communications," IEE Proceedings, vol. 152, no. 2, pp. 97-102, 2005.

[14] A. Locquet, S. Ortin, V. Udaltsov et al., "Delay-time identification in chaotic optical systems with two delays," in Semiconductor Lasers and Laser Dynamics II, vol. 6184 of Proceedings of SPIE, Strasbourg, France, April 2006.

[15] R. M. Nguimdo, M. C. Soriano, and P. Colet, "Role of the phase in the identification of delay time in semiconductor lasers with optical feedback," Optics Letters, vol. 36, no. 22, pp. 4332-4334, 2011.

[16] M. C. Soriano, L. Zunino, O. A. Rosso, I. Fischer, and C. R. Mirasso, "Time scales of a chaotic semiconductor laser with optical feedback under the lens of a permutation information analysis," IEEE Journal of Quantum Electronics, vol. 47, no. 2, pp. 252-261, 2011.

[17] Y.-C. Wang, J.-S. Liang, A.-B. Wang, and M.-J. Zhang, “Timedelay extraction in chaotic laser diode using RF spectrum analyser," Electronics Letters, vol. 46, no. 24, pp. 1621-1623, 2010.

[18] Y. Wu, Y. C. Wang, P. Li, A.-B. Wang, and M.-J. Zhang, "Can fixed time delay signature be concealed in chaotic semiconductor laser with optical feedback?" IEEE Journal of Quantum Electronics, vol. 48, no. 11, pp. 1371-1379, 2012.

[19] J.-G. Wu, Z.-M. Wu, X. Tang et al., "Simultaneous generation of two sets of time delay signature eliminated chaotic signals by using mutually coupled semiconductor lasers," IEEE Photonics Technology Letters, vol. 23, no. 12, pp. 759-761, 2011.

[20] N. Q. Li, W. Pan, S. Y. Xiang et al., "Photonic generation of wideband time-delay-signature-eliminated chaotic signals utilizing an optically injected semiconductor laser," IEEE Photonics Technology Letters, vol. 48, no. 10, pp. 1339-1345, 2012.

[21] N. Q. Li, W. Pan, S. Y. Xiang, L. S. Yan, B. Luo, and X. H. Zou, "Loss of time delay signature in broadband cascade-coupled semiconductor lasers," IEEE Photonics Technology Letters, vol. 24, no. 23, pp. 2187-2190, 2012.

[22] A. P. A. Fischer, O. K. Andersen, M. Yousefi, S. Stolte, and D. Lenstra, "Experimental and theoretical study of filtered optical feedback in a semiconductor laser," IEEE Journal of Quantum Electronics, vol. 36, no. 3, pp. 375-384, 2000.

[23] A. P. A. Fischer, M. Yousefi, D. Lenstra, M. W. Carter, and G. Vemuri, "Filtered optical feedback induced frequency dynamics in semiconductor lasers," Physical Review Letters, vol. 92, no. 2, Article ID 023901, 4 pages, 2004.

[24] H. Erzgräber and B. Krauskopf, "Dynamics of a filteredfeedback laser: influence of the filter width," Optics Letters, vol. 32, no. 16, pp. 2441-2443, 2007. 


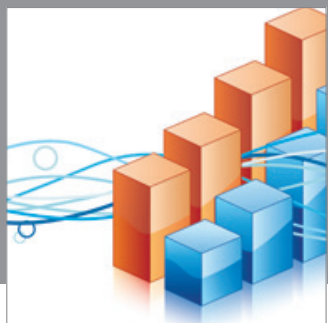

Advances in

Operations Research

mansans

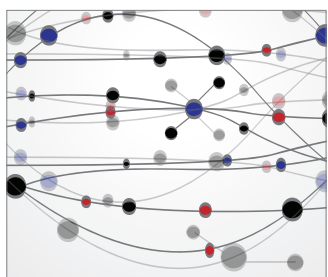

The Scientific World Journal
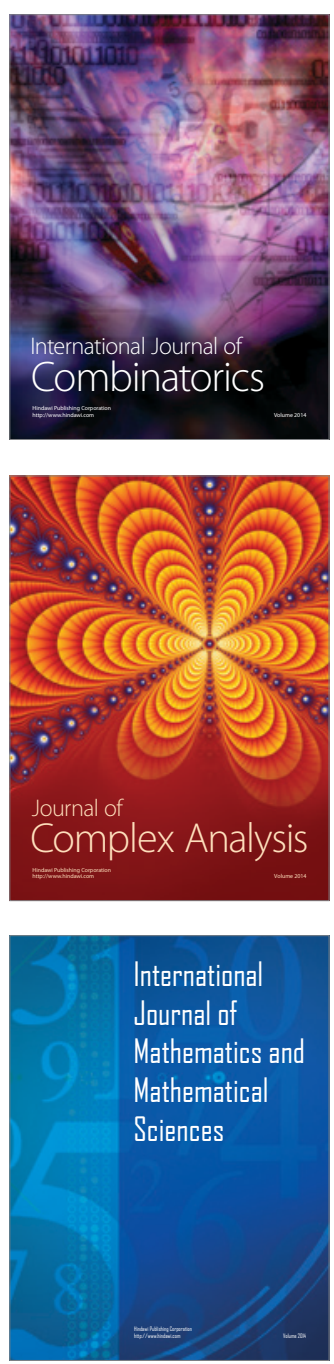
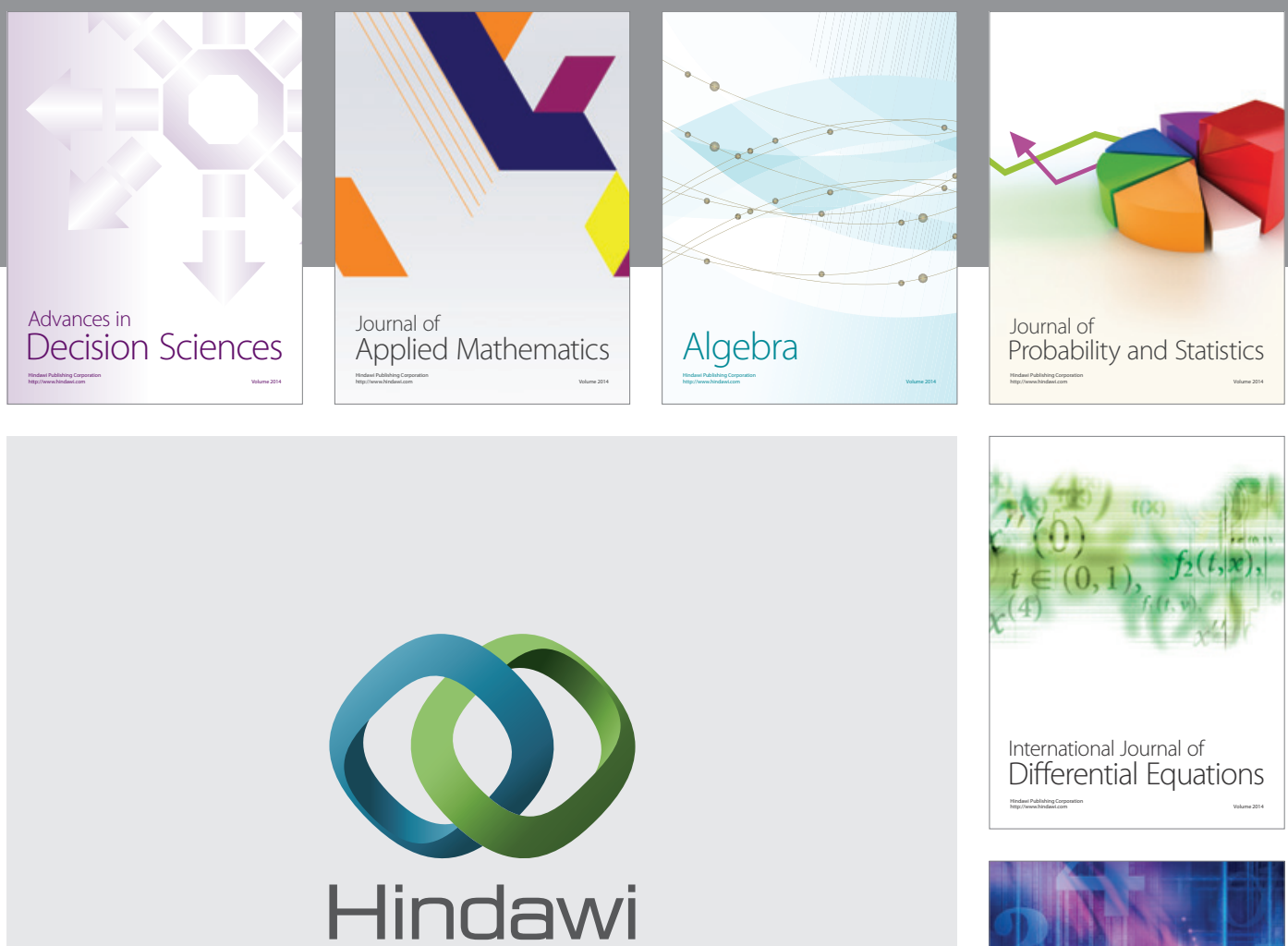

Submit your manuscripts at http://www.hindawi.com
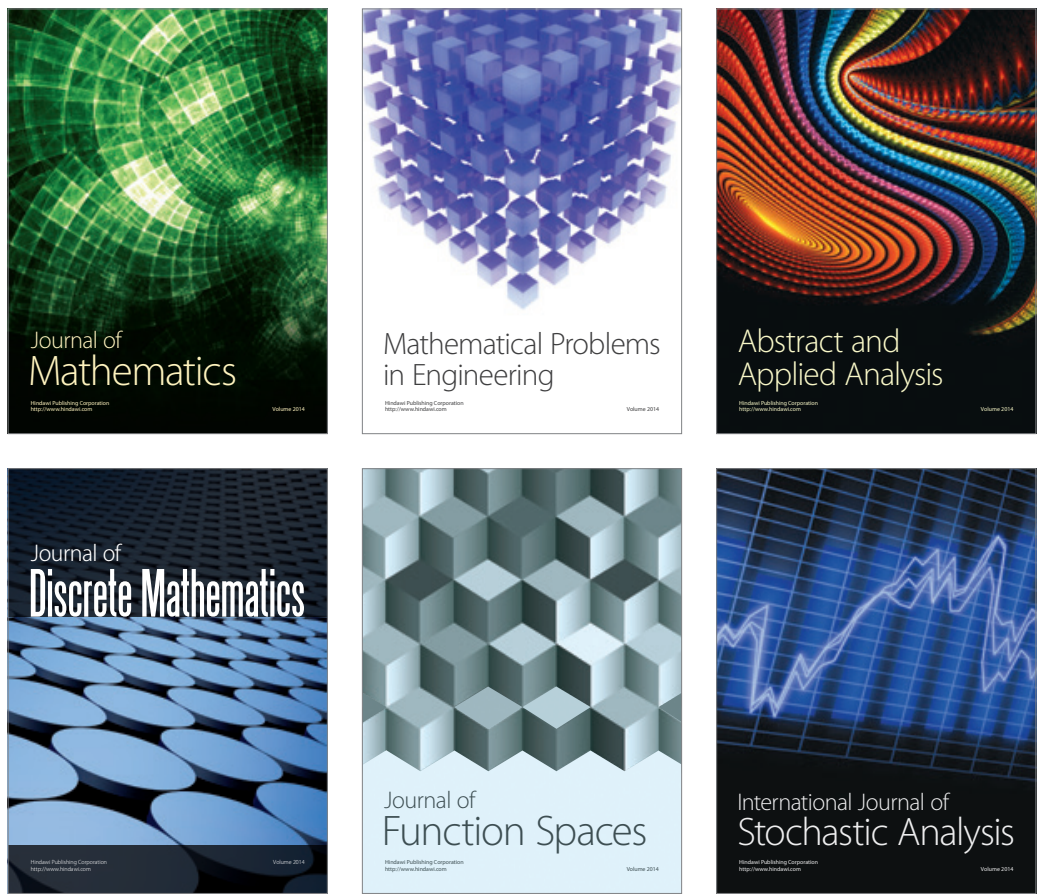

Journal of

Function Spaces

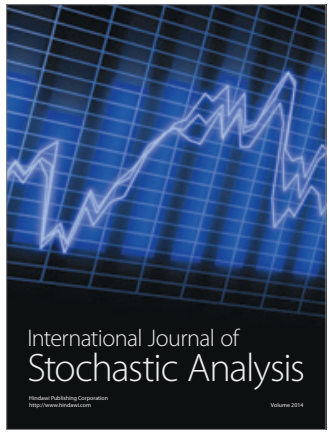

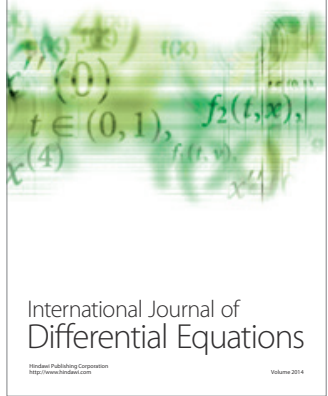
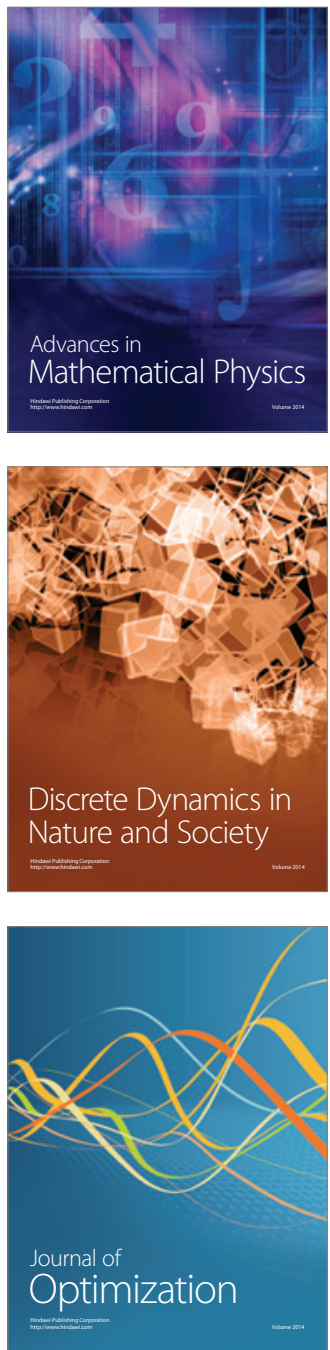